\title{
Economic Modeling of Syndromic Surveillance Systems - A Roundtable Discussion on Association of State and Territorial Health Official's (ASTHO) Investment Decision Model
}

\author{
Paula Soper ${ }^{1}$, Scott Gordon 1 , Marcus Rennick ${ }^{1}$, Jane Blake*2, Victoria Adams², Taylor \\ Hemby², Walter (Chip) Jansen $^{2}$, Nasim Moghadam² and KC Decker ${ }^{2}$
}

${ }^{1}$ Association of State and Territorial Health Officials, Arlington, VA, USA; ${ }^{2}$ Booz Allen Hamilton, McLean, VA, USA

\section{Objective}

Provide a demonstration of the recently developed prototype decision analysis model for syndromic surveillance investments. The roundtable will be used to discuss the model, obtain feedback on its usefulness, and brainstorm future uses and improvements.

\section{Introduction}

One of ASTHO's key goals is to help its jurisdictions meet member needs for technical assistance, including making informed decisions about their syndromic surveillance options. To help them make such decisions, ASTHO worked with Booz Allen to create a decision analysis model, which factors in both a Value of Information (VOI) model and a Return on Investment (ROI) model. The model provides a dashboard of its outputs, which is a simple, easy-to-understand comparative view of multiple syndromic surveillance investment scenarios.

\section{Description}

The roundtable will include a demonstration of the decision model, a review of how it can be used in practice, and a facilitated discussion covering its usefulness, applicability in the US and internationally, and potential improvements for the future. The roundtable will be moderated by subject matter expert panel members who participated in the model creation, an ASTHO staff member who facilitated development, and the Booz Allen project manager.

Facilitators will include:

- Jane Blake (Booz Allen)

- Marcus Rennick (ASTHO)

- Bryant Karras (university of Washington)

- David Buckeridge (McGill University)

\section{Audience Engagement}

Facilitators will begin by providing a demonstration of the model, following by polling the audience to provide scenarios to run the model. The poll will include questions around parameters including cost, functionality, granularity, breadth, and timeliness for an as-is and two potential future scenarios. After the demo, the facilitators will review results, ask for feedback, and discuss potential gaps and additional uses.

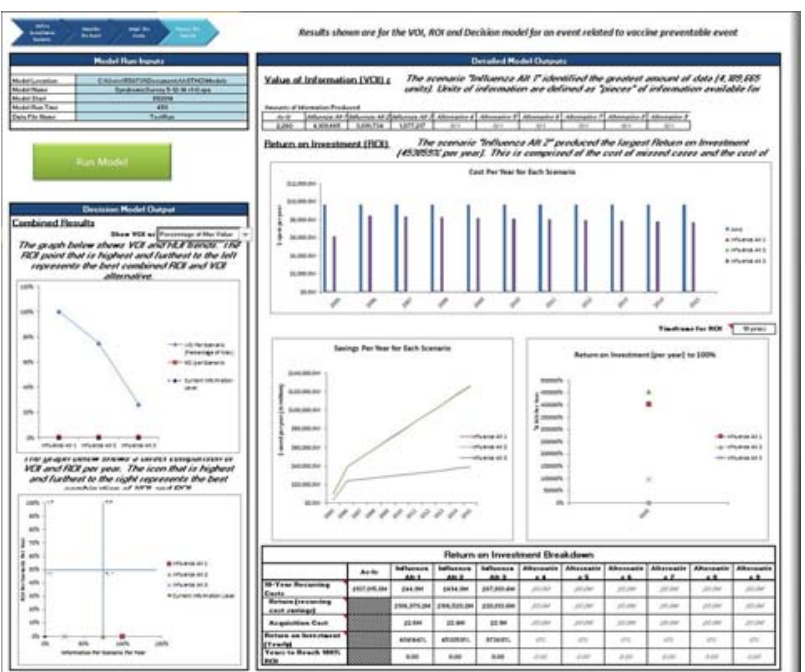

Keywords

Return on Investment; Value of Information; Syndromic Surveillance; Decision Model; Analytics

\section{Acknowledgments}

Timothy Andrews, Booz Allen Hamilton

Atar Baer, Seattle-King County Public Health

David Buckeridge, Montreal Public Health and Quebec Public Health Institute

Jim Collins, Michigan Department of Community Health

Julia Gunn, Boston Public Health Commission

Bryant Karras, Washington Public Health State

Aaron Kite-Powell, Armed Forces Health Surveillance Center

Joe Lombardo, Johns Hopkins University

Vishal Pachigar, Booz Allen Hamilton

\section{*Jane Blake}

E-mail: Blake_Jane@bah.com 